\title{
An Adaptive Control Approach for Discrete-Time Systems with Unknown Backlash at the Input
}

T.S. SANTOS ${ }^{1}$, Departamento de Telemática, FEEC, UNICAMP, Cx.P. 6101, 13083-970 Campinas, SP, Brasil.

F.H.T. VIEIRA ${ }^{2}$ Escola de Engenharia Elétrica e de Computação, Universidade Federal de Goiás, Av. Universitária, n. 1488, Quadra 86, Bloco A, $3^{\circ}$ piso, 74605-010 Setor Leste Universitário, Goiânia, GO, Brasil.

\begin{abstract}
In this paper, we focus on the development of an adaptive control scheme for systems with unknown backlash. More specifically, we propose a discretetime adaptive backlash inverse based controller for plants that have a known linear part and an unknown backlash at its input. We also demonstrate that the harmful effects of backlash can be cancelled by adding two control blocks. These blocks are the following: an adaptive backlash inverse structure and an adaptive linear filter controller. The latter block provides a new adaptive law for the updating of the backlash parameters. We show by simulations that our adaptive control approach improves the system performance once the difference between the backlash output and the adaptive backlash inverse input, converges to a very small value. The simulations involve a third order system that highlights the efficiency of the proposed adaptive control approach.
\end{abstract}

Keywords. Backlash, Adaptive Control, Linear Adaptive Controller.

\section{Introduction}

Backlash nonlinearities are common in control system components, such as, mechanical connections and electromagnetic devices with hysteresis [15], [10], [8], [9]. The undesirable effects of backlash are the main factors of severely limiting the performance of feedback systems. These undesirable effects consist of non-differentiable nonlinearities and include the decrease of static output accuracy, poor transient performance, limit cycles and instability [12].

A commonly used approach of cancelling the harmful effects of nonlinearities is the implementation of their inverse characteristics into the controller structure. Notice that this technique of cancelling of harmful effects can be applied to discretetime models with backlash [13], [14].

Adaptive systems are inherently nonlinear in general. Their behaviors are therefore quite complex, making them difficult to be analyzed. Much work remains before

\footnotetext{
${ }^{1}$ talia@dt.fee.unicamp.br

${ }^{2}$ flavio@eee.ufg.br
} 
a reasonably complete, coherent theory be available for such systems. Due to the complex behavior of adaptive systems, it is necessary to consider several aspects of them [1], [4], depending on the considered circumstances.

For the development of our work, adaptive control techniques are useful and important tools once they can be used to recognize the nonlinearities as well as to compensate their harmful effects. The application of adaptive controllers in system processing nonlinearities can simplify the component specifications of the system, reduce the total system cost and increase its reliability [5], [7].

In this paper, we develop a novel adaptive inverse control approach in discretetime. We propose an adaptive control scheme consisting of an adaptive backlash inverse and an adaptive filter for a controller structure. The backlash inverse allows that the undesirable effects of the nonlinearity be cancelled when considering its exact parameters. Then, it is important to minimize the control error of the system, that is, the difference between the backlash output and the adaptive inverse backlash input in order to eliminate the damaging effects caused by backlash.

A wide variety of recursive algorithms has been developed in the literature for the training of adaptive filters and linear controllers [11], [2]. The operation of an adaptive filtering algorithm involves two basic procedural steps: a filtering procedure designed to produce an output in response to a sequence of input data and an adaptive procedure, whose function is to provide a mechanism for the adaptive control of an adjustable set of parameters used in the filtering procedure [5]. In fact, in this work we aim at including a basic adaptive linear filter block (proportional controller) to enhance the adaptive controlling of the backlash inverse parameters.

In [15], the authors present a controller structure to compensate the backlash effects in continuous-time. In this same work, an update law for the estimation of the backlash parameters are suggested. In our adaptive control approach, we consider a feedback block to compensate the damaging caused by backlash. This feedback block consists of a linear adaptive controller that provides a new update law for the estimation of the backlash parameters.

The paper is organized as follows: in Section 2 we present the backlash compensation, that is, the backlash model and its inverse in discrete-time and also the adaptive backlash inverse. In Section 3, we introduce the adaptive backlash inverse control model, the controller structure and the proposed adaptive parameter training. Finally in Section 4, we analyze the performance of our adaptive control approach through a numerical example and in Section 5, we present the conclusions of this work.

\section{Backlash Compensation}

\subsection{Backlash model}

The backlash model considered in this paper as well as a simple mechanical connection example taken from [15] are both presented in Figure 1.

In the backlash characteristic shown in Figure 1.a, $v(t)$ is the input, $u(t)$ is the output, and $c_{r}>0$ is the right "crossing" on the $v$-axis, while $c_{l}<0$ is the left "crossing" on the $v$-axis [16]. 


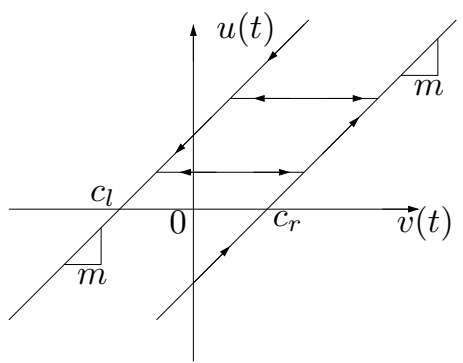

(a)

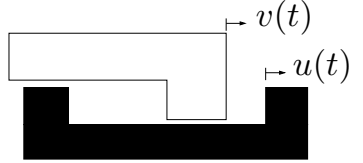

(b)

Figure 1: (a) Backlash model; (b) Mechanical connection.

The discrete-time model of the backlash is given by:

$$
u(t)=\left\{\begin{array}{l}
m\left(v(t)-c_{l}\right) \text { if } v(t) \leq v_{l}=\frac{u(t-1)}{m}+c_{l} \\
m\left(v(t)-c_{r}\right) \text { if } v(t) \geq v_{r}=\frac{u(t-1)}{m}+c_{r} \\
u(t-1) \text { otherwise }
\end{array}\right.
$$

where the values $v_{l}$ and $v_{r}$ are the $v$-axis projections of the intersections of the two parallel lines of slope $m$ with the horizontal inner segment containing $u(t-1)$ [13].

Equation (2.1) is the so-called friction driven hysteresis backlash model, i.e., the driven member retains its position when the backlash gap is not closed, as if kept in place by strong friction [16]. It can be verified that (2.1) is a piecewise-linear dynamical system with three distinct regions of behavior, here called upward active, downward active and gap regions.

\subsection{Backlash inverse model}

The desired function of a backlash inverse is to cancel the harmful effects of backlash on system performance: the delay corresponding to the time needed to traverse an inner segment of $B\left({ }^{\cdot}\right)$ and the information loss occurring on an inner segment when the output $u(t)$ remains constant while the input $v(t)$ continues is changed. That is, given a desired signal $u_{d}(t)$ for $u(t)$, a backlash inverse $B I\left({ }^{\cdot}\right)$ is such that $u_{d}(t)=B\left(B I\left(u_{d}(t)\right)\right)$ (Figure 2).

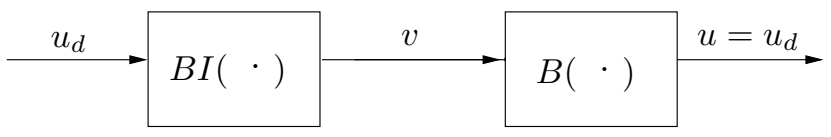

Figure 2: Inverting a backlash.

The discrete-time model of the backlash inverse is represented by the following mapping:

$$
v(t)=\left\{\begin{array}{l}
v(t-1) \text { if } u_{d}(t)=u_{d}(t-1) \\
\frac{u_{d}(t)}{m}+c_{l} \text { if } u_{d}(t)<u_{d}(t-1) \\
\frac{u_{d}(t)}{m}+c_{r} \text { if } u_{d}(t)>u_{d}(t-1)
\end{array}\right.
$$


Notice that the formulation of the backlash inverse in discrete-time do not make use of the derivatives of $u_{d}(t)$. Another advantage of the discrete-time formulation over the continuous-time one is that closed-loop signal bounds can be established even for the case of different slopes $m[16]$.

\subsection{Adaptive backlash inverse}

The backlash inverse $B I(\cdot)$ defined by $(2.2)$ can be approximated by replacing the vertical jumps between its upward and downward lines by continuous curves with bounded gains. For example, a vertical transition is replaced with a line segment which links two sides of $B I(\cdot)$ and has a slope of a positive and finite value.

For an adaptive backlash inverse $B I\left({ }^{\cdot}\right)$, we replace the backlash parameters $m, c_{r}$ and $c_{l}$ by their estimates $\hat{m}, \hat{c_{r}}$ and $\hat{c_{l}}$ that are obtained from the adaptive update law as the following:

$$
v(t)=\left\{\begin{array}{l}
v(t-1) \text { if } u_{d}(t)=u_{d}(t-1) \\
\frac{u_{d}(t)}{\hat{m}}+\hat{c_{l}} \text { if } u_{d}(t)<u_{d}(t-1) \\
\frac{u_{d}(t)}{\hat{m}}+\hat{c_{r}} \text { if } u_{d}(t)>u_{d}(t-1)
\end{array}\right.
$$

In the next section, we use an adaptive backlash inverse $\hat{B} I(\cdot)$ as part of the proposed adaptive control algorithm for plants with an unknown backlash $B(\cdot)$.

\section{Adaptive Backlash Inverse Control}

We consider a discrete plant with a linear part $G(D)$ and a backlash nonlinearity $B(\cdot)$ at its input

$$
y(t)=G(D) u(t), u(t)=B[v(t)]
$$

where $G(D)=K_{p} \frac{Z(D)}{P(D)}$ with $K_{p}$ being a nonzero constant gain and monic polynomials denoted by $Z(D)$ and $P(D)$. Here the symbol $D$ is used to denote a z-transform variable or an advance operator. In our control problem, the input $u(t)$ to the linear plant is not accessible for measurement, and the backlash $B\left({ }^{\cdot}\right)$ is unknown while the linear part $G(D)$ is known. Our objective is to design a feedback control $v(t)$ using an adaptive linear controller parameter $(f)$ to achieve the stabilization and a close match of the system response to the desired one.

Our control strategy consists of including a linear adaptive controller with gain parameter $f$ in the controller structure to cancel the harmful effects of backlash and to decrease the control error in an adaptive environment, i. e., we adaptively compute the backlash parameters. Therefore, the adaptive linear controller $f$ has an important role in our control scheme.

\subsection{The controller structure}

A controller structure that compensates the backlash effects in continuous-time can be found in [15]. We follow the same structure configuration of that paper, that is, a feedback control $v(t)$ that uses the measurement plant output $y(t)$ to achieve global 
stabilization and close tracking of a reference signal $y_{m}(t)$ by the measurement plant output $y(t)$.

Similarly to the controller used in [15], in the reference [3], the author included one more feedback block control. The controllers proposed in [15] and [3] are both adaptive but they differ in the involved time consideration. The first one is in continuous-time and the second one is in discrete-time. In this paper we work in a discrete-time environment. Thus, the examples given in [3] are appropriate and will be considered in Section 4.

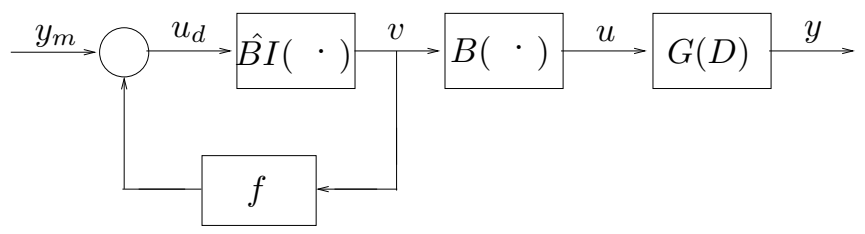

Figure 3: Adaptive backlash inverse controller structure.

Different from [3], in this work we add an unique feedback block (adaptive linear controller) to compensate the damage caused by backlash. Figure 3 shows our adaptive backlash inverse controller structure, in which the desired control signal $u_{d}(t)$ is generated by:

$$
u_{d}(t)=y_{m}(t)+f v(t)
$$

where $y_{m}(t)$ is the reference signal and $f$ is the adaptive linear controller parameter.

Substituting (2.3) into (3.2), we have:

$$
u_{d}(t)=\left\{\begin{array}{l}
y_{m}+f v(t-1) \text { if } u_{d}(t)=u_{d}(t-1) \\
y_{m}+f \frac{u_{d}(t)}{\hat{m}}+\hat{c_{l}} \text { if } u_{d}(t)<u_{d}(t-1) \\
y_{m}+f \frac{u_{d}(t)}{\hat{m}}+\hat{c_{r}} \text { if } u_{d}(t)>u_{d}(t-1)
\end{array}\right.
$$

Many control problems can be solved by using techniques based on the mean square error minimization [17]. For example, one can obtain the adaptive filter parameters or estimate some parameters of a feedback control system through the least-mean-square (LMS) algorithm [17], [6].

In our problem, the control error is defined as:

$$
e(t)=u(t)-u_{d}(t)
$$

That is, our aim is to cancel the backlash undesirable effects using its inverse as shown in Figure 2. Let $J$ be the performance control function given in terms of the mean squared error as follows:

$$
J=E\left\{e^{2}(t)\right\}
$$

where $E\{\cdot\}$ denotes the expectation operator.

Now, by substituting the equation (3.4) into (3.5) we can write the performance control function $J$ as:

$$
J=E\left\{\left(u(t)-u_{d}(t)\right)^{2}\right\}
$$


In the next subsection, we describe how the adaptive linear control parameter $f$ and the backlash inverse parameters are updated in our proposal.

\subsection{Adaptive laws}

As described earlier, we define a performance function $J$ that is related to the elimination of the undesirable backlash effects when applying an adaptive backlash inverse.

In this section, we find the adaptive rules to update our control scheme parameters. Our objective is to minimize $J$, that is, to reduce the difference between $u(t)$ and $u_{d}(t)$. To this end, we deduce the partial derivatives in relation of the control parameters and make them equal to zero, as presented below:

$$
\begin{aligned}
& \frac{\partial J}{\partial f}=0 \\
& \frac{\partial J}{\partial \hat{m}}=0 \\
& \frac{\partial J}{\partial \hat{c_{r}}}=0 \\
& \frac{\partial J}{\partial \hat{c_{l}}}=0
\end{aligned}
$$

The above equations allow us to obtain the adaptive linear parameter in (3.7) and the backlash inverse estimated parameters $\hat{m}, \hat{c_{r}}, \hat{c_{l}}$ in (3.8), (3.9) and (3.10), respectively. More explicitly, the updating equations for the parameters $f, \hat{m}, \hat{c_{r}}$, $\hat{c_{l}}$ are expressed as:

$$
f=\frac{\mathcal{F}_{n}}{\mathcal{F}_{d}}, \quad \hat{m}=\frac{\mathcal{M}_{n}}{\mathcal{M}_{d}}, \quad \hat{c_{r}}=\frac{\mathcal{C}_{1 n}}{\mathcal{C}_{1 d}}, \quad \hat{c_{l}}=\frac{\mathcal{C}_{2 n}}{\mathcal{C}_{2 d}}
$$

where

$$
\begin{aligned}
\mathcal{F}_{n} & =u y_{m} \hat{m}^{2}+u \hat{w} \hat{m}^{3}-y_{m}^{2} \hat{m}^{2}-y_{m} \hat{m}^{3} \hat{w} \\
\mathcal{F}_{d} & =u y_{m} \hat{m}+y_{m} \hat{w} \hat{m}^{2}+\hat{w}^{2} \hat{m}^{3}+u \hat{w} \hat{m}^{2} \\
\mathcal{M}_{n} & =u y_{m} f^{2}+u f^{3} \hat{w} \\
\mathcal{M}_{d} & =u y_{m} f+u f^{2} \hat{w}-y_{m}^{2} f-2 y_{m} f^{2} \hat{w}-f^{3} \hat{w}^{2} \\
\mathcal{C}_{1 n} & =u f \hat{m}^{2}-u f^{2} \hat{m}-y_{m} \hat{m}^{2} f \\
\mathcal{C}_{1 d} & =f^{2} \hat{m}^{2} \\
\mathcal{C}_{2 n} & =u f \hat{m}^{2}-u f^{2} \hat{m}-y_{m} \hat{m}^{2} f \\
\mathcal{C}_{2 d} & =f^{2} \hat{m}^{2}
\end{aligned}
$$

We included a new parameter to simplify the notation of the updating parameter equations. This parameter is: $\hat{w}$. The parameter $\hat{w}$ corresponds to a choice between $\hat{c_{r}}$ and $\hat{c_{l}}$. The values of the parameter $\hat{w}$ depend on the backlash region. If the 
backlash region is in the upward active region, this parameter will be: $\hat{w}=\hat{c_{r}}$, else if it is in the downward active region it will be: $\hat{w}=\hat{c_{l}}$. Otherwise, if the backlash region is in the gap region, we assume the same previous values for the parameters $\hat{w}$.

\section{Numerical Example}

In this section, we follow the same illustrative example presented in [3]. The example consists of controlling a third order system.

The third order linear plant is

$$
G(D)=\frac{1 \times 10^{-5} z^{2}+4 \times 10^{-5} z+1 \times 10^{-5}}{z^{3}-2.88504 z^{2}+2.77196 z-0.88692}
$$

with unknown $B(\cdot)$ :

$$
m=1.3, c_{r}=3, c_{l}=-3
$$

We adopted the following configuration set for the closed-loop system: $y_{m}=$ $10 \sin 12.6 t$, and the initial values equal to: $\hat{m}=0.696, \hat{c_{r}}=0.5, \hat{c_{l}}=-0.5$ and filter parameter $f=0.22$. The difference between the backlash $B(\cdot)$ parameters and their inverse $\hat{B I}(\cdot)$ parameters are depicted in Figure 4.

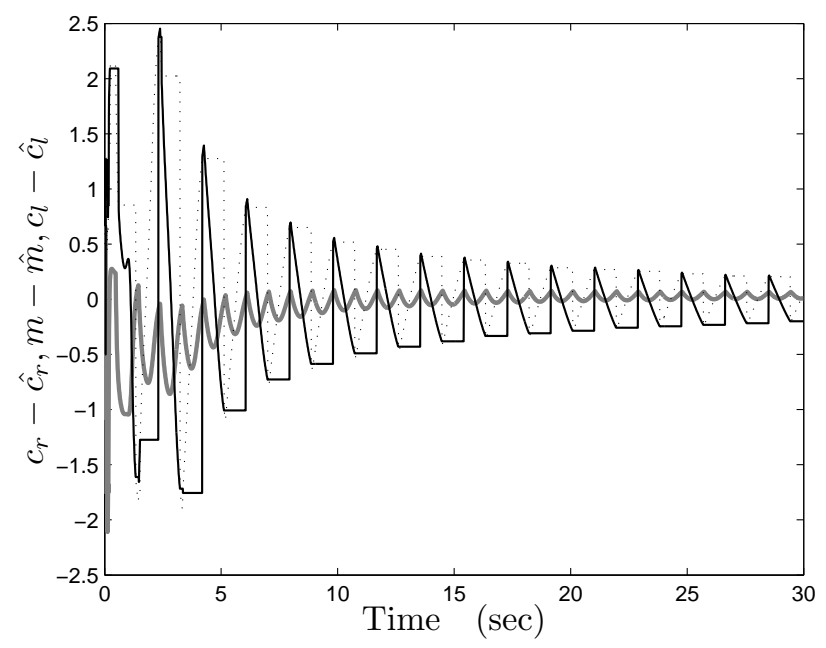

Figure 4: The difference between the backlash parameters and their estimated parameters $c_{r}-\hat{c_{r}}$ (black dotted), $m-\hat{m}$ (thick gray solid), $c_{l}-\hat{c_{l}}$ (black solid).

Figure 5 compares the control error results obtained by using the approach described in [3] and our control error results (thick black solid curve). It can be observed that the control errors given by our adaptive control approach are similar to those given by [3]. As a consequence, our approach is more suitable for adaptive systems due to its lower computational complexity. This lower computational 
complexity can be easily verified once, different from [3], our proposal involves the insertion of only one feedback block.

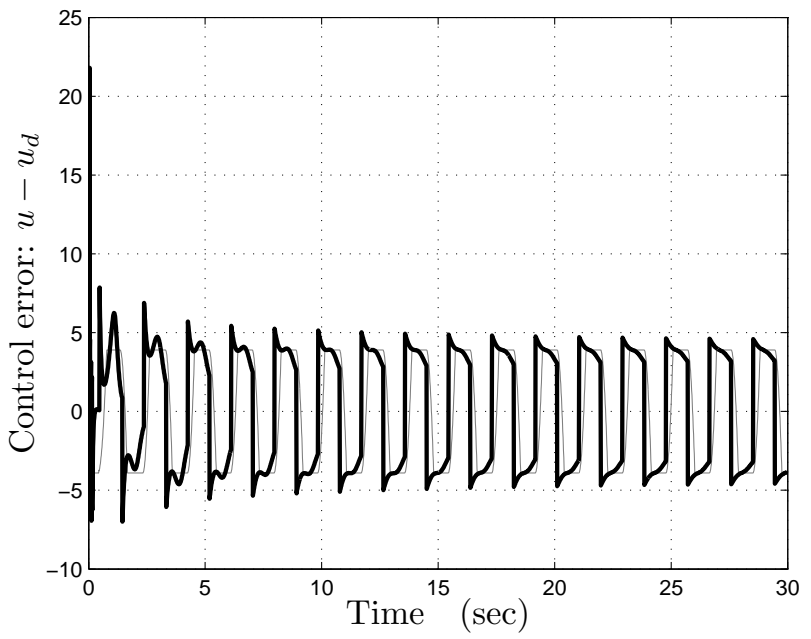

Figure 5: Control error of [3] (gray solid) and ours (thick black solid).

In this example, one can see that the backlash inverse parameters approximately reach the same values of the backlash parameters, once the difference between them converges to zero.

\section{Conclusion}

We have developed a discrete-time adaptive backlash inverse based controller for plants which have a known linear part and an unknown backlash at its input. Our controller structure is composed of an adaptive linear proportional controller and an adaptive backlash inverse to cancel the harmful effects of backlash.

We verified through simulations that the backlash inverse parameter values reach the backlash parameter values in a short time interval. Moreover, we obtained a satisfactory control error compared to the errors achieved with more complex algorithms such as that presented in [3], for example.

It is relevant to comment that in the approach of [3], it was added three additional structures to compensate the backlash effects. On the other hand, in this paper we used only one additional block (adaptive proportional controller block) maintaining the control error at lower levels. That is, our control approach, besides reaching high efficiency, it requires less computational effort. These characteristics make the proposed control scheme attractive for applications that demand fast processing (e.g., real-time applications). Therefore, we can conclude that this work provides an important contribution for discrete-time systems with unknown backlash. 
As a future work, we intend to extrapolate our control scheme to the continuoustime case. However, in this case, it is necessary to take into account the derivatives of $u_{d}(t)$.

\section{Acknowledgment}

We would like to thank the CNPq for the financial support, Prof. Dr. Luiz Henrique Bonani do Nascimento and MSc. Grace Silva Deaecto for the help in the preparation of this manuscript and Prof. Dr. Basílio Ernesto de Almeida Milani, Prof. Dr. Gang Tao (University of Virginia) and Prof. Dr. Alessandra Dutra Coelho for the important comments given to us.

Resumo. Neste artigo, focamos no desenvolvimento de um esquema de controle adaptativo para sistemas com folga desconhecida. Mais especificamente, propomos um controlador adaptativo baseado na (função) inversa da folga para plantas discretas no tempo que tem como entrada uma parte linear e uma folga desconhecida. Demonstramos também que os efeitos prejudiciais da folga podem ser cancelados adicionando dois blocos de controle. Estes blocos são os seguintes: uma estrutura inversa da folga e um filtro controlador linear adaptativo. O segundo bloco mencionado fornece novas regras adaptativas para a atualização adaptativa dos parâmetros da folga inversa. Através de simulações, mostramos que nossa proposta de controle adaptativo melhora o desempenho do sistema uma vez que a diferença entre a saída da folga e a entrada da folga inversa adaptativa converge para um valor baixo. As simulações envolvem um sistema de terceira ordem a fim de verificar a eficiência do algoritmo proposto de controle adaptativo.

\section{References}

[1] K.J. Astrom, B. Wittenmark, "Adaptive Control", Addison-Wesley, New York, 1989.

[2] M.G. Bellanger, "Adaptive Digital Filters", Marcel Dekker, New York, 2001.

[3] A.D. Coelho, "Controle Adaptativo para Sistemas com Backlash", Master Thesis, Escola Federal de Engenharia de Itajubá, 1997.

[4] G. Feng, R. Lozano, "Adaptive Control Systems", Newnes - Reed Educ. Profes. Publishing Ltd, New York, 1999.

[5] M. Grundelius, D. Angeli, Adaptive control of system with backlash acting on the input, in "Proceedings of 35th Conference on Decision and Control", Kobe, Japan, 1996.

[6] S. Haykin, "Adaptive Filter Theory", Prentice Hall, Englewood Cliffs, 1991.

[7] P.A. Ioannou, K.S. Tsakalis, A robust direct adaptive controller, IEEE Transactions on Automatic Control, AC-31, No. 11 (1986), 1033-1043.

[8] B.E.A. Milani, Conjuntos de confinamento final uniforme para sistemas lineares com folgas nos controles, in "Congresso Brasileiro de Automática", pp. 601-606, 2006 . 
[9] B.E.A. Milani, Nonlinear static feedback stabilization of linear system with backlash, in "Proceedings of 46th Conference on Decision and Control", New Orleans, pp. 1716-1721, 2007.

[10] M. Nordin, P.O. Gutman, Controlling mechanical systems with backlash - a survey, Automatica, 38 (2002), 1633-1649.

[11] S. Sastry, M. Bodson, "Adaptive Control Stability, Convergence and Robustness", Prentice Hall, Englewood Cliffs, 1989.

[12] J.J.E. Slotine, W. Li, "Applied Nonlinear Control", Prentice Hall, Englewood Cliffs, 1991.

[13] G. Tao, P.V. Kokotovic, Adaptive control of systems with backlash, Automatica, 29, No. 2 (1993), 323-335.

[14] G. Tao, P.V. Kokotovic, Adaptive control of systems with unknown output backlash", IEEE Transactions on Automatic Control, 40, No. 2 (1995), 326330 .

[15] G. Tao, P.V. Kokotovic, Continuous-time adaptative control of systems with unknown backlash, IEEE Transactions on Automatic Control, 40, No. 6 (1995), 1083-1087.

[16] G. Tao, P.V. Kokotovic, "Adaptive Control of Systems with Actuator and Sensor Nonlinearities", John Wiley \& Sons, New York, 1996.

[17] J.R. Treichler, C.R. Johnson Jr., M.G. Larimore, "Theory and Design of Adaptive Filters", John Wiley \& Sons, 1987. 